\title{
Multi-Robot Manipulation with no Communication Using Only Local Measurements
}

\author{
Zijian Wang $^{1}$ and Mac Schwager ${ }^{2}$
}

\begin{abstract}
This paper presents a novel approach to coordinate the manipulation forces of a group of robots without explicit communication during a cooperative manipulation task. Robots use the measurements of the motion of the object as the only information to reach a consensus on their forces. It is proven that the consensus can be reached even if all the robots have different velocity and acceleration measurements since they take measurements at different attachment points around the object while the object is rotating and translating. The convergence of the leader-following process where a leader robot actively steers the forces of all follower robots to navigate the object along a desired trajectory is also proven with Lyapunov stability arguments. We verify our method in both numerical simulations and a physics simulator, where we transport a grand piano with 1001 robots.
\end{abstract}

\section{INTRODUCTION}

In this paper we present a scheme for a very large number of small, simple robots to transport a comparatively large object through a specified trajectory towards a destination. In our previous work [1], it was proven that this cooperative manipulation task could be accomplished without explicit communication. This is highly advantageous for large groups of simple robots, for which a wireless communication network would be overly power hungry, computationally demanding, and algorithmically complex. Instead, using the measurements of the motion of the object itself, each robot can indirectly get some information about the forces from all the other robots, since the motion of the object is related to the sum of the forces applied by the group of robots according to Newton's second law. It is shown that this information is enough for robots to reach a consensus on their forces without communication. A leader robot or a human operator then guides the transport of the object along a desired trajectory while all other follower robots reinforce the leader's force through the force consensus.

One critical assumption in our previous work [1] is that every robot needs to measure the velocity and acceleration at the center of mass of the object. In practice this requires that the robots have some global information, e.g. they know their attachment points and the geometry of the object, so they can compute the motion of the center of mass from their local motion. Moreover, even if the robots know where the center of mass is, computing its motion from local measurements may introduce errors. Therefore, in this paper we relax this global measurement assumption by allowing each robot to only measure the local velocity

*This work was supported in part by NSF grant CNS-1330036.

${ }^{1} \mathrm{Z}$. Wang is with the Department of Mechanical Engineering, Boston University, Boston, MA 02215, USA, z jwang abu . edu

${ }^{2} \mathrm{M}$. Schwager is with the Department of Mechanical Engineering and the Division of Systems Engineering, Boston University, Boston, MA 02215, USA, schwagerabu . edu

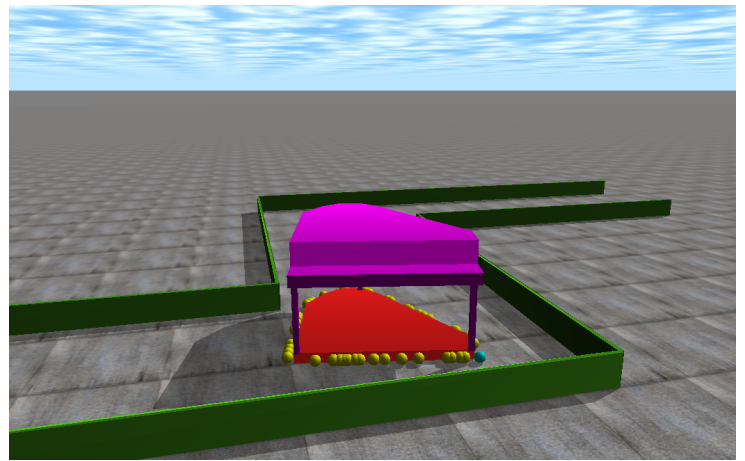

Fig. 1. An example of multi-robot manipulation. A group of tiny robots (denoted by spheres around the bottom of the grand piano) grasp the base platform, where a grand piano is mounted, and apply forces to move the large piano together to the destination.

and acceleration at its attachment point on the object. Since the object may rotate and translate, different robots attached at different points may sense quite different local velocities and accelerations. However, in this paper we prove that, using only the local measurements, the force consensus will still be achieved under a simple condition on the geometry and mass properties of the object. This result extends the applicability of our approach to real-life situations, for example, transporting large assemblies such as aircraft parts or building components in a manufacturing or construction site, removing debris in a disaster site, or retrieving incapacitated human survivors after a disaster.

Our work is related to multi-agent consensus in control community and multi-robot manipulation in the robotics community. Consensus algorithms [2], [3] have been studied intensively recently, and provide a way for multiple agents to agree on a common value by only communicating with neighboring agents. This common value can also be dynamically controlled by introducing a leader agent [4], and the controllability of this leader-following dynamics has been studied [5]. We take this as a tool to coordinate the forces of a group of robots. However, unreliable communication may undermine the consensus process in a large network due to the dropped and noisy data, package collision and delays [6], [7]. We therefore propose a novel way in this paper to achieve force consensus without explicit communication by measuring the motion of the object as an indirect way to pass information. This idea of using indirect sensing has also been found in the behavior of ants [8], where it was pointed out that ants coordinate themselves during collective transport by detecting small-scale vibration or deformation of the object, rather than communicating with each other directly. 
In the field of robotic manipulation, the seminal work [9] studied how to control the force on the end-effector of a manipulator, which can be used to address the assumption in our paper that each robot can apply an accurate force to the object. As for the specific topic of multi-robot manipulation, [10] is among the earliest attempts to discuss different coordination strategies with different amounts of sensing and communication. Later on, a popular solution to cooperative manipulation is called caging [11], [12], [13], where a group of robots surround the object to make sure that the object cannot escape or slip away when the robots are moving. Recently, manipulation experiments were done with more than 100 robots using ensemble control techniques [14]. The work [15] proposed a kinematics-based motion controller for multiple transporter robots according to their relative position to the centroid of the object. A simple yet effective multi-robot manipulation approach that also uses no communication was demonstrated in [16], where robots utilized the visual occlusion to push a tall object towards destination. [17] measured the forces exerted by real ants during collective food retrieval and proposed a hybrid model for robots to replicate ants' behaviors. Beyond ground robots, there is also work that use multiple aerial vehicles, to collectively manipulate large objects [18]. Our work, in contrast to all the paper above, is fully decentralized, uses no communication and guarantee the coordination of robots through force consensus.

The rest of the paper is organized as follows. In Section II, we set up the multi-robot manipulation problem, first reviewing the formulation from our previous work. Section III proves that the force consensus can be achieved when robots only have local velocity and acceleration measurements. In Section IV we consider the performance of the system when a leader (a more powerful robot, or a human operator) directs the motion of the object along a desired trajectory. In Section $\mathrm{V}$ we briefly analyze how the system will be affected if a symmetry assumption is violated. Finally, simulation results are demonstrated in Section VI.

\section{Problem Formulation}

In this section we first summarize the problem set up and main results from our previous work [1] and then formulate our problem for the case where robots only sense the object motion locally, at their attachment point.

\section{A. Background and Preliminaries}

In this section, we will briefly introduce the set up of our problem from our previous work [1]. We consider a manipulation task in a $2 \mathrm{D}$ region $Q \subset \mathbb{R}^{2}$, as shown in Figure 2. The objective is to transport an object with mass $M$ and moment of inertia $J$ along a desired trajectory to a goal location. The object can translate and rotate, and we denote the velocity and acceleration at the center of the object as $v_{c}$ and $a_{c}$, respectively, while the angular velocity is denoted by $\omega$. Two forces from the environment are modeled: the gravity $M g$ and the viscous friction $\mu_{v} v_{c}$. We do not account for static friction in this paper, as we assume either the object sits on top of the robots, which then act as a smart pallet, or the object is already on a wheeled pallet of some sort which is pushed by the robots.

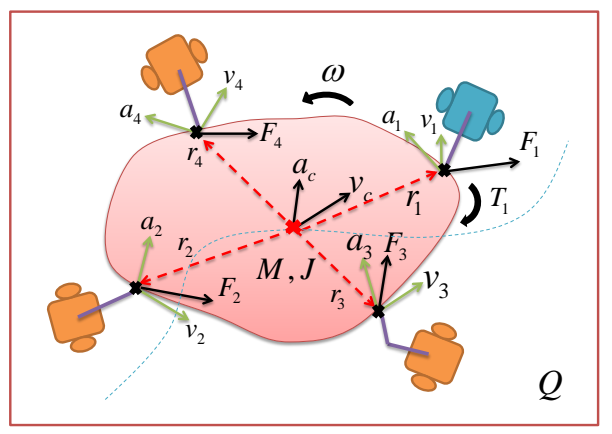

Fig. 2. A schematic of multi-robot manipulation. Each robot $i$ can contribute to the manipulation by applying a force $F_{i}$ to the object. The force coordination can be achieved without communication by measuring either $v_{c}$ and $a_{c}$ at the center of mass [1] or local $v_{i}$ and $a_{i}$, which is proven in this paper.

We have a fleet of $N$ robots, each of which has some manipulation mechanism to grasp the object and apply a $2 \mathrm{D}$ force, $F_{i}=\left(f_{i x}, f_{i y}\right)^{T}, i \in\{1,2, \cdots, N\}$, to the object. Then the translational dynamics of the object can be written as

$$
M a_{c}=\sum_{i=1}^{N} F_{i}-\mu_{v} v_{c} .
$$

In addition to applying a force, each robot can also measure the linear velocity and acceleration of the object. In our previous work, we made the assumption that each robot knows the velocity and acceleration at the center of mass of the object. It was shown that this information is enough for all robots to align their forces in the same direction with the same magnitude (i.e. attain a force consensus) without communication. This is achieved by the following force control law for each robot,

$$
\begin{aligned}
\dot{F}_{i} & =\sum_{j=1, j \neq 1}^{N}\left(F_{j}-F_{i}\right)=\sum_{j=1}^{N} F_{j}-N F_{i} \\
& =M a_{c}+\mu_{v} v_{c}-N F_{i} .
\end{aligned}
$$

Remarkably, equation (2) is an implementation of a well known consensus law [2] and will lead to all robots applying equal force vectors to the object. One might expect that each robot $i$ needs to communicate with its neighbors to get $F_{j}$. However the sum of all unknown $F_{j}$ plus robot $i$ 's own force is a known quantity by (1) given that robots can measure $a_{c}$ and $v_{c}$. Hence the consensus control law can be computed using (3) without communication.

A leader (which can be a robot or a human operator), indexed as robot 1 , is assigned to steer the consensus value such that the total force from the group of robots can be controlled. We assume that the leader robot is more powerful than other follower robots in that the leader knows where the specified trajectory is relative to the object while the followers have no global navigation information. We also assume that the leader can measure the angular velocity of the object $\omega$ and apply a torque, $T_{1}$, to the object. We showed in [1] that under a symmetry assumption (Assumption 1 below), 
the rotational dynamics of the object can be simplified as

$$
J \alpha=J \dot{\omega}=T_{1}+\sum_{i=1}^{N} r_{i} \times F_{i}-\frac{\mu_{v}}{M} J \omega,
$$

where $r_{i}=x_{i}-x_{c}$ is the vector pointing from the center of mass to the attachment point of robot $i$ in the global reference frame. We also developed a controller for the leader robot to actively guide the group to move the object to follow the trajectory with the rotation being controlled. For more details, please refer to our previous paper [1].

\section{B. Using Measurements at the Attachment Points}

In this paper, we explore the possibility of using the measurement at each robot's attachment point to reach a consensus on the forces. We start the problem by comparing the difference between the measurement at the center of mass and the attachment point, which is revealed by the following equations,

$$
\begin{gathered}
v_{i}=v_{c}+\omega \times r_{i}, \\
a_{i}=\dot{v}_{i}=a_{c}+\alpha \times r_{i}+\omega \times\left(\omega \times r_{i}\right),
\end{gathered}
$$

where $v_{i}$ and $a_{i}$ are the velocity and acceleration, respectively, of the object measured by each robot at its own attachment point. The term, $\omega \times\left(\omega \times r_{i}\right)$, is the centrifugal acceleration. Note that the Coriolis acceleration in our case is zero since the attachment point remains stationary in the object's local reference frame. As a result, one can see that although we analyze the forces in the global reference frame, it makes no difference when the robots take measurements and apply forces in their local reference frames.

We wish to continue to use the naive force updating law (3), but to substitute $v_{c}$ and $a_{c}$ by $v_{i}$ and $a_{i}$, respectively. We can write the new force control law as

$$
\dot{F}_{i}=M a_{i}+\mu_{v} v_{i}-N F_{i} .
$$

In the following sections, we prove that the control law (6) is able to drive all the forces to a consensus under certain conditions without explicit communication among robots.

\section{FORCE COORDINATION WITHOUT LEADER}

In this section we study the convergence of the forces applied by the robots under the force control law (6). The goal is for the robots to align their forces to a common force vector, i.e. to reach a consensus on their forces.

\section{A. Matrix Representation}

We first put the dynamics of the object and the robots' forces in a matrix form which is more amenable to analysis. Using (1), (4), and (5), we can rewrite (6) as

$$
\begin{aligned}
\dot{F}_{i}= & M\left(a_{c}+\alpha \times r_{i}+\omega \times\left(\omega \times r_{i}\right)\right)+ \\
& \mu_{v}\left(v_{c}+\omega \times r_{i}\right)-N F_{i} \\
= & M a_{c}+\mu_{v} v_{c}-N F_{i}+M \omega \times\left(\omega \times r_{i}\right)+\mu_{v} \omega \times r_{i}+ \\
& M\left(\frac{1}{J} \sum_{i=1}^{N} r_{i} \times F_{i}-\frac{\mu_{v}}{M} \omega\right) \times r_{i} \\
= & \left(\sum_{j=1}^{N} F_{j}-N F_{i}\right)+\frac{M}{J}\left(\sum_{j=1}^{N} r_{j} \times F_{j}\right) \times r_{i}+ \\
& M \omega \times\left(\omega \times r_{i}\right),
\end{aligned}
$$

where the first term in (7) by itself would lead to a consensus, as it is the same as in (2). The second and third terms in (7) appear as disturbances, denoting the additional effects caused by the rotation in the tangential and centrifugal directions. We initially make the following assumption, which will be relaxed in Section V.

Assumption 1: (Centrosymmetric) The robots' attachment points are centrosymmetric around the center of mass of the object, meaning that for any robot $i$, there exists another robot $j \neq i$ such that $r_{i}=-r_{j}$.

Under this assumption, the centrifugal term $M \omega \times\left(\omega \times r_{i}\right)$ in (7) will not have any influence on the consensus because it points towards the center of mass and will be canceled out by the paired symmetric robots. Hence, we only need to study the first two terms in (7), as follows

$$
\dot{F}_{i}=\left(\sum_{j=1}^{N} F_{j}-N F_{i}\right)-\frac{M}{J} r_{i} \times\left(\sum_{j=1}^{N} r_{j} \times F_{j}\right) .
$$

In order to investigate the overall behavior of $N$ robots rather than one individual robot in (8), we can stack all the forces into one column vector and rewrite (8) in matrix form. The cross product can be dealt with using skew symmetric matrices. The resulting matrix version of (8) can be written

$$
\dot{F}^{+}=\left(-L_{a}^{+}-\frac{M}{J} R_{a}^{+}(t)\right) F^{+},
$$

where $F^{+}=\left(f_{1 x}, f_{1 y}, 0, f_{2 x}, f_{2 y}, 0, \cdots, f_{N x}, f_{N y}, 0\right)^{T}$ is a vector containing augmented $3 \mathrm{D}$ forces with $0 \mathrm{z}$-axis components. $L_{a}^{+}=\left(L_{i j}^{+}\right)_{3 N \times 3 N},\{i, j\} \in\{1,2, \cdots, N\}$, where

$$
L_{i j}^{+}=\left\{\begin{array}{ccc}
\left(\begin{array}{ccc}
N-1 & 0 & 0 \\
0 & N-1 & 0 \\
0 & 0 & 0
\end{array}\right) & \text { if } i=j \\
\left(\begin{array}{ccc}
-1 & 0 & 0 \\
0 & -1 & 0 \\
0 & 0 & 0
\end{array}\right) & \text { if } i \neq j
\end{array}\right.
$$

is an extended graph Laplacian matrix for the 3D case. $R_{a}^{+}(t)=\left(R_{i}^{+}(t) R_{j}^{+}(t)\right)_{3 N \times 3 N}$ is a collection of products of skew symmetric matrices, and

$$
R_{i}^{+}(t)=\left(\begin{array}{ccc}
0 & 0 & r_{i y} \\
0 & 0 & -r_{i x} \\
-r_{i y} & r_{i x} & 0
\end{array}\right),
$$

where $r_{i x}$ and $r_{i y}$ represent the subcomponent of $r_{i}$ in the $x$ and $y$ axes, respectively. Note that $R_{a}^{+}(t)$ is time-varying since $r_{i}$ changes while the object is rotating.

Notice that both $L_{a}^{+}$and $R_{a}^{+}(t)$ are sparse because of the zero elements that we introduced when lifting the $2 \mathrm{D}$ system to $3 \mathrm{D}$ in order to express the cross products as skew symmetric matrices. It will be helpful if we can eliminate the sparsity and only focus on the $x$ and $y$ dimensions. Consider every entry in the big $R_{a}^{+}(t)$ matrix,

$$
R_{i}^{+}(t) R_{j}^{+}(t)=\left(\begin{array}{ccc}
-r_{i y} r_{j y} & r_{i y} r_{j x} & 0 \\
r_{i x} r_{j y} & -r_{i x} r_{j x} & 0 \\
0 & 0 & -r_{i y} r_{j y}-r_{i x} r_{j x}
\end{array}\right) .
$$


Since $F_{j}^{+}=\left(f_{j x}, f_{j y}, 0\right)$, if we calculate $R_{i}^{+}(t) R_{j}^{+}(t) F_{j}^{+}$, then the term $-r_{i y} r_{j y}-r_{i x} r_{j x}$ in (10) will be multiplied by zero and will disappear in the product. So we can eliminate the sparsity by removing the zero lines in $F^{+}, L_{a}^{+}$and removing the third column and third row in $R_{i}^{+}(t) R_{j}^{+}(t)$. Using this fact, (9) can be further simplified as

$$
\dot{F}=\left(-L_{a}-\frac{M}{J} R_{a}(t)\right) F
$$

where $F=\left(f_{1 x}, f_{1 y}, \cdots, f_{N x}, f_{N y}\right)^{T} \cdot L_{a}=\left(L_{i j}\right)_{2 N \times 2 N}$, where

$$
L_{i j}= \begin{cases}\left(\begin{array}{cc}
N-1 & 0 \\
0 & N-1
\end{array}\right) & \text { if } i=j \\
\left(\begin{array}{cc}
-1 & 0 \\
0 & -1
\end{array}\right) & \text { if } i \neq j\end{cases}
$$

and $R_{a}(t)=\left(R_{i j}(t)\right)_{2 N \times 2 N}$, where

$$
R_{i j}(t)=\left(\begin{array}{cc}
-r_{i y} r_{j y} & r_{i y} r_{j x} \\
-r_{i x} r_{j y} & -r_{i x} r_{j x}
\end{array}\right) .
$$

The expression (11) gives the dynamics of all the robots' forces in the system, and is the main subject of study of the rest of the paper. The consensus of all the forces can be determined by investigating the equilibria and stability of (11), as described in the following sections.

\section{B. Time-independent Characterization of $R_{a}(t)$}

The difficulty of analyzing (11) lies in the time-varying nature of $R_{a}(t)$, making the entire system time-varying. Here we prove, surprisingly, that the rank and eigenvalues of $R_{a}(t)$ are time-invariant.

Proposition 1: The rank of $R_{a}(t)$ is one, and the single nonzero eigenvalue of $R_{a}(t)$ is a constant $\lambda_{\min }\left(R_{a}(t)\right)=$ $-\sum_{i=1}^{N}\left\|r_{i}\right\|^{2}$.

Proof: Denote $r_{i}$ using polar coordinates, $r_{i}=$ $\left(r_{i x}, r_{i y}\right)=\left(\left\|r_{i}\right\| \cos \left(\theta+\theta_{i}\right),\left\|r_{i}\right\| \sin \left(\theta+\theta_{i}\right)\right)$, where $\theta$ is the angle of the object, which changes over time, and $\theta_{i}$ is the constant angle of $r_{i}$ in the object's reference frame. Then we have

$$
\begin{aligned}
& R_{i j}(t)= \\
& \left\|r_{i}\right\|\left\|r_{j}\right\|\left(\begin{array}{cc}
-\sin \left(\theta+\theta_{i}\right) \sin \left(\theta+\theta_{j}\right) & \sin \left(\theta+\theta_{i}\right) \cos \left(\theta+\theta_{j}\right) \\
\cos \left(\theta+\theta_{i}\right) \sin \left(\theta+\theta_{j}\right) & -\cos \left(\theta+\theta_{i}\right) \cos \left(\theta+\theta_{j}\right)
\end{array}\right) .
\end{aligned}
$$

The first row of $R_{a}(t)$ is

$$
\begin{gathered}
R_{a}(1,:)=\left\|r_{1}\right\| \sin \left(\theta+\theta_{1}\right)\left(-\left\|r_{1}\right\| \sin \left(\theta+\theta_{1}\right),\right. \\
\left\|r_{1}\right\| \cos \left(\theta+\theta_{1}\right), \cdots,-\left\|r_{N}\right\| \sin \left(\theta+\theta_{N}\right), \\
\left.\left\|r_{N}\right\| \cos \left(\theta+\theta_{N}\right)\right)
\end{gathered}
$$

By observation, every row in $R_{a}(t)$ is linearly dependent on the first row because the $(2 k-1)$-th and $(2 k)$-th row, $k \in\{1,2, \cdots, N\}$, of $R_{a}(t)$ can be written as

$$
R_{a}(2 k-1,:)=\frac{\left\|r_{k}\right\| \sin \left(\theta+\theta_{k}\right)}{\left\|r_{1}\right\| \sin \left(\theta+\theta_{1}\right)} R_{a}(1,:),
$$

$$
R_{a}(2 k,:)=\frac{-\left\|r_{k}\right\| \cos \left(\theta+\theta_{k}\right)}{\left\|r_{1}\right\| \sin \left(\theta+\theta_{1}\right)} R_{a}(1,:) .
$$

Therefore, $\operatorname{rank}\left(R_{a}(t)\right)=1$ for any $t$.

Since the rank of $R_{a}(t)$ is one, it has only one nonzero eigenvalue. In order to find this eigenvalue, let us first look at its element, $R_{i j}(t)$. By solving $\left|\lambda I_{2 \times 2}-R_{i j}(t)\right|=0$ we can get the the eigenvalues and corresponding eigenvectors of $R_{i j}(t)$ :

$$
\begin{gathered}
\lambda_{1}=0, v_{1}=\left(\cos \left(\theta+\theta_{j}\right), \sin \left(\theta+\theta_{j}\right)\right)^{T} \\
\lambda_{2}=-\left\|r_{i}\right\|\left\|r_{j}\right\| \cos \left(\theta_{i}-\theta_{j}\right), \\
v_{2}=\left(-\sin \left(\theta+\theta_{i}\right), \cos \left(\theta+\theta_{i}\right)\right)^{T}
\end{gathered}
$$

We can make several observations: the eigenvalues of $R_{i j}(t)$ remain constant regardless of the rotation of the object; the eigenvectors of $R_{i j}(t)$ rotate as the object rotates; $v_{1}$ is a unit vector along $r_{j}$; the direction of $v_{2}$ can be acquired by rotating $r_{i}$ clockwise by $\pi / 2$ radians.

Denote $v_{2}=\left(-\sin \left(\theta+\theta_{i}\right), \cos \left(\theta+\theta_{i}\right)\right)^{T}$ as $e_{i}$, which is the unit eigenvector associated with the non-zero eigenvalue of $R_{i j}(t)$. Then in general we have $R_{i j}(t) e_{i}=$ $-\left\|r_{i}\right\|\left\|r_{j}\right\| \cos \left(\theta_{i}-\theta_{j}\right) e_{i}$, and $R_{i j}(t) r_{j}=0$. Also it is not hard to verify that $R_{i j}(t) e_{j}=-\left\|r_{i}\right\|\left\|r_{j}\right\| e_{i}$. Then we can construct a vector $e_{a}=\left(\left\|r_{1}\right\| e_{1},\left\|r_{2}\right\| e_{2}, \cdots,\left\|r_{N}\right\| e_{N}\right)^{T}$, which turns out to be the eigenvector of $R_{a}(t)$ because

$$
\begin{aligned}
R_{a}(t) e_{a} & =\left(\begin{array}{cccc}
R_{11} & R_{12} & \cdots & R_{1 N} \\
R_{21} & R_{22} & \cdots & R_{2 N} \\
\vdots & \vdots & \cdots & \vdots \\
R_{N 1} & R_{N 2} & \cdots & R_{N N}
\end{array}\right)\left(\begin{array}{c}
\left\|r_{1}\right\| e_{1} \\
\left\|r_{2}\right\| e_{2} \\
\vdots \\
\left\|r_{N}\right\| e_{N}
\end{array}\right) \\
& =-\sum_{i=1}^{N}\left\|r_{i}\right\|^{2}\left(\begin{array}{c}
\left\|r_{1}\right\| e_{1} \\
\left\|r_{2}\right\| e_{2} \\
\vdots \\
\left\|r_{N}\right\| e_{N}
\end{array}\right) .
\end{aligned}
$$

Hence, the only one nonzero eigenvalue of $R_{a}(t)$ is $\lambda_{a}=$ $-\sum_{i=1}^{N}\left\|r_{i}\right\|^{2}$, for any $t$.

\section{Consensus Analysis}

Now that we have obtained enough understanding of the time-varying term $R_{a}(t)$, we can continue to study the combined effect of $\left(L_{a}-\frac{M}{J} R_{a}(t)\right)$ in (11). We start our analysis by looking at the equilibria of (11), and then we find the range of the eigenvalues of $\left(L_{a}-\frac{M}{J} R_{a}(t)\right)$. Note that (11) is a time-varying system, whose stability cannot be completely characterized by the positions of the eigenvalues. As a result, we apply Barbalat's lemma to prove that (11) will asymptotically converge to the consensus.

Under the centrosymmetric assumption (Assumption 1), $\left(L_{a}-\frac{M}{J} R_{a}(t)\right)$ has two time-invariant eigenvectors associated with zero eigenvalues,

$$
\mathbf{1}_{x}=\left(\begin{array}{c}
1 \\
0 \\
1 \\
0 \\
\vdots \\
1 \\
0
\end{array}\right)_{2 N \times 1}, \mathbf{1}_{y}=\left(\begin{array}{c}
0 \\
1 \\
0 \\
1 \\
\vdots \\
0 \\
1
\end{array}\right)_{2 N \times 1} .
$$


The reason is as follows. First of all, from the properties of the Laplacian matrix we know that $L_{a} \mathbf{1}_{x}=L_{a} \mathbf{1}_{y}=$ 0 . Secondly, according to our centrosymmetric assumption, for $\forall i, \exists j \neq i$, s.t. $r_{i}+r_{j}=0$, or alternatively, $\left\|r_{i}\right\| \cos \left(\theta+\theta_{i}\right)+\left\|r_{j}\right\| \cos \left(\theta+\theta_{j}\right)=0$ and $\left\|r_{i}\right\| \sin \left(\theta+\theta_{i}\right)+$ $\left\|r_{j}\right\| \sin \left(\theta+\theta_{j}\right)=0$. Вy (15), $R_{a}(1,:) \mathbf{1}_{x}=\left\|r_{1}\right\| \sin (\theta+$ $\left.\theta_{1}\right) \sum_{i=1}^{N}\left(-\sin \left(\theta+\theta_{i}\right)\right)=0, R_{a}(1,:) \mathbf{1}_{y}=\left\|r_{1}\right\| \sin (\theta+$ $\left.\theta_{1}\right) \sum_{i=1}^{N}\left(\cos \left(\theta+\theta_{i}\right)\right)=0$. Due to the fact that all rows of $R_{a}(t)$ are linearly dependent on the first row, we have $\left(-L_{a}-\frac{M}{J} R_{a}(t)\right) \mathbf{1}_{x}=0,\left(-L_{a}-\frac{M}{J} R_{a}(t)\right) \mathbf{1}_{y}=0$.

Moreover, we know that $s_{x} \mathbf{1}_{x}+s_{y} \mathbf{1}_{y}, s_{x}, s_{y} \in \mathbb{R}$ are the time-invariant equilibria of (11) since they are the solutions to $\dot{F}=0$. These equilibria are exactly the force consensus that we are looking to achieve, since all forces will have the same $x$ and $y$ components. Our goal is to prove that the system will finally converge to these equilibia, which is shown in the following proposition and theorem.

Proposition 2: Under the centrosymmetric assumption, the eigenvalues of $\left(-L_{a}-\frac{M}{J} R_{a}(t)\right)$ are less than or equal to zero if

$$
\frac{M}{J} \sum_{i=1}^{N}\left\|r_{i}\right\|^{2}<N
$$

Proof: The eigenvalue of the sum of two matrices can be bounded by Weyl's theorem [19], which is a result for Hermitian matrices. $L_{a}$ is obviously a real symmetric matrix, and therefore it is Hermitian. Since $R_{a}(t)=\left(R_{i j}(t)\right)$, and

$$
\begin{aligned}
& R_{j i}(t)= \\
& \left\|r_{i}\right\|\left\|r_{j}\right\|\left(\begin{array}{cc}
-\sin \left(\theta+\theta_{j}\right) \sin \left(\theta+\theta_{i}\right) & \sin \left(\theta+\theta_{j}\right) \cos \left(\theta+\theta_{i}\right) \\
\cos \left(\theta+\theta_{j}\right) \sin \left(\theta+\theta_{i}\right) & -\cos \left(\theta+\theta_{j}\right) \cos \left(\theta+\theta_{i}\right)
\end{array}\right) .
\end{aligned}
$$

By comparing with (14) we have $R_{i j}(t)=\left(R_{j i}(t)\right)^{T}$. This implies that $R_{a}(t)$ is also a real symmetric (and therefore Hermitian) matrix.

Arrange the eigenvalues of $-L_{a}$ and $-\frac{M}{J} R_{a}(t)$ in increasing order,

$$
\begin{aligned}
& \lambda_{1}\left(-L_{a}^{-}\right) \rightarrow \lambda_{2 N}\left(-L_{a}^{-}\right): \\
&-N \leqslant-N \leqslant \cdots \leqslant-N \leqslant 0 \leqslant 0 \\
& \lambda_{1}\left(-\frac{M}{J} R_{a}^{-}\right) \rightarrow \lambda_{2 N}\left(-\frac{M}{J} R_{a}^{-}\right): \\
& 0 \leqslant 0 \leqslant \cdots \leqslant 0 \leqslant \frac{M}{J} \sum_{i=1}^{N}\left\|r_{i}\right\|^{2} .
\end{aligned}
$$

If we also arrange the eigenvalues of $-L_{a}-\frac{M}{J} R_{a}(t)$ in increasing order, then according to Weyl's,

$$
\begin{aligned}
\lambda_{2 N-2}\left(-L_{a}-\frac{M}{J} R_{a}(t)\right) & \leqslant \lambda_{2 N-2}\left(-L_{a}\right)+\lambda_{2 N}\left(-\frac{M}{J} R_{a}(t)\right) \\
& \leqslant-N+\frac{M}{J} \sum_{i=1}^{N}\left\|r_{i}\right\|^{2} .
\end{aligned}
$$

If $\frac{M}{J} \sum_{i=1}^{N}\left\|r_{i}\right\|^{2}<N$, then

$$
\lambda_{2 N-2}\left(-L_{a}-\frac{M}{J} R_{a}(t)\right)<0,
$$

which means that the third largest eigenvalue of $-L_{a}-$ $\frac{M}{J} R_{a}(t)$ is less than zero. We also know that $-L_{a}-\frac{M}{J} R_{a}(t)$ has two eigenvalues at zero, so $\lambda_{2 N-1}\left(-L_{a}-\frac{M}{J} R_{a}(t)\right)=$ $\lambda_{2 N}\left(-L_{a}-\frac{M}{J} R_{a}(t)\right)=0$. In summary, $-L_{a}(t)-\frac{M}{J} R_{a}(t)$ has two zero eigenvalues with $\mathbf{1}_{x}, \mathbf{1}_{y}$ as the eigenvectors, and all other eigenvalues are negative.

Theorem 1: Under the centrosymmetric assumption (Assumption 1), (11) will reach a consensus on all forces if (18) is satisfied. The consensus value is the the average of all the initial forces.

Proof: We can decompose the force as,

$$
F=s_{x} \mathbf{1}_{x}+s_{y} \mathbf{1}_{y}+\delta, s_{x}, s_{y} \in \mathbb{R},
$$

where $\left(s_{x} \mathbf{1}_{x}+s_{y} \mathbf{1}_{y}\right)$ is the state of consensus and $\delta$ is the group disagreement vector [2]. Then we have

$$
\begin{aligned}
\dot{F}=\dot{\delta} & =\left(-L_{a}-\frac{M}{J} R_{a}(t)\right)\left(s_{x} \mathbf{1}_{x}+s_{y} \mathbf{1}_{y}+\delta\right) \\
& =\left(-L_{a}-\frac{M}{J} R_{a}(t)\right) \delta .
\end{aligned}
$$

Knowing the dynamics of the disagreement vector, we can choose the Lyapunov function to be

$$
V=\frac{1}{2} \delta^{T} \delta
$$

Then according to Proposition 2 we have

$$
\begin{gathered}
\dot{V}=\delta^{T} \dot{\delta}=\delta^{T}\left(-L_{a}-\frac{M}{J} R_{a}(t)\right) \delta \\
\leqslant \lambda_{2 N}\left(-L_{a}-\frac{M}{J} R_{a}(t)\right)\|\delta\|^{2} \leqslant 0 \\
\ddot{V}=2 \delta^{T}\left(-L_{a}-\frac{M}{J} R_{a}(t)\right)^{2} \delta+\delta^{T}\left(-\frac{M}{J} \dot{R}_{a}(t)\right) \delta .
\end{gathered}
$$

We can show that $\ddot{V}$ is bounded because: (i) $\delta$ is bounded since $V=\frac{1}{2} \delta^{T} \delta$ and $\dot{V} \leqslant 0$. (ii) $L_{a}$ and $R_{a}(t)$ are apparently bounded. (iii) $\dot{R}_{a}(t)$ is related to the angular velocity, $\omega$, of the object. Note that the dynamics of $\omega$ is given by (4), which is a bounded-input bounded-output (BIBO) system. Since $\delta$ is bounded, we have $F$ is bounded, then $\omega$ is bounded and finally $\dot{R}_{a}(t)$ is also bounded. According to Barbalat's, $V \geqslant 0, \dot{V} \leqslant 0$ plus $\ddot{V}$ bounded will ensure that $\dot{V} \rightarrow 0$, i.e., $\delta$ will converge asymptotically to the invariant set $\Omega$, where

$$
\Omega=\left\{\delta \mid \delta=p_{x} \mathbf{1}_{x}+p_{y} \mathbf{1}_{y}, p_{x}, p_{y} \in \mathbb{R}\right\} .
$$

Therefore, $F$ in (11) will converge to its equilibria, $\left(s_{x}+\right.$ $\left.p_{x}\right) \mathbf{1}_{x}+\left(s_{y}+p_{y}\right) \mathbf{1}_{y}, s_{x}, s_{y}, p_{x}, p_{y} \in \mathbb{R}$, which is the state of consensus since all the forces will be equal. Finally, from (7) we know $\sum_{i=1}^{N} \dot{F}_{i}=0$, meaning that the total force of the group is conserved during the entire process. Hence $F$ will converge to the average of all the initial forces.

\section{Group Force Control via Leader Following}

In real applications, we also want to steer the consensus force so that we can control the motion of the object. This steering process is done by introducing a leader robot (or a human operator) who chooses its own force such that all other followers converge to, and therefore reinforce the effect of, the leader's force. 
Suppose that robot 1 is the leader robot, and define $\tilde{L}(t)=$ $\left(L_{a}+\frac{M}{J} R_{a}(t)\right)$. As proposed in [20], we can separate the leader and followers in $\tilde{L}(t)$ as

$$
\tilde{L}(t)=\left[\begin{array}{cc}
\tilde{L}_{l}(t) & \tilde{L}_{f l}^{T}(t) \\
\tilde{L}_{f l}(t) & \tilde{L}_{f}(t)
\end{array}\right],
$$

where $\tilde{L}_{l}(t) \in \mathbb{R}^{2 \times 2}, \quad \tilde{L}_{f}(t) \in \mathbb{R}^{(2 N-2) \times(2 N-2)}$ and $\tilde{L}_{f l}(t) \in \mathbb{R}^{(2 N-2) \times 2}$. Now we can rewrite (11) in the sense of leader-following:

$$
\dot{F}_{f}=-\tilde{L}_{f}(t) F_{f}-\tilde{L}_{f l}(t) F_{1},
$$

where $F_{f} \in \mathbb{R}^{2 N-2}$ is the stacked force vector of all follower robots and $F_{1} \in \mathbb{R}^{2}$ is the leader's force. The objective here is to show that given the leader's force $F_{1}$, every follower robot's force $F_{i}, i=\{2,3, \cdots, N\}$, will converge to $F_{1}$. This is verified through the following proposition and theorem.

Proposition 3: $\tilde{L}_{f}(t)$ has full rank under the centrosymmetric assumption.

Proof: From Proposition 2 we know that $\tilde{L}(t)$ has $2 N-$ 2 non-zero eigenvalues, so $\operatorname{rank}(\tilde{L}(t))=2 N-2$, meaning that $\tilde{L}(t)$ has $2 N-2$ linearly independent rows (and columns) and 2 linearly dependent rows (and columns). $\tilde{L}_{f}(t)$ can be obtained by removing the first two rows and columns of $\tilde{L}(t)$. Denote the $i$-th row and $j$-th column of $\tilde{L}(t)$ by $\tilde{L}(i$, : ) and $\tilde{L}(:, j)$. Then by (12), (15), (16), and (17) we have $\tilde{L}(1,:)=-(\tilde{L}(3,:)+\tilde{L}(5,:)+\cdots+\tilde{L}(2 N-1,:)), \tilde{L}(2,:$ )$=-(\tilde{L}(4,:)+\tilde{L}(6,:)+\cdots+\tilde{L}(2 N,:)), \tilde{L}(:, 1)=-(\tilde{L}(:$ $, 3)+\tilde{L}(:, 5)+\cdots+\tilde{L}(:, 2 N-1)), \tilde{L}(:, 2)=-(\tilde{L}(:, 4)+\tilde{L}(:$ $, 6)+\cdots+\tilde{L}(:, 2 N))$. In other words, the first two rows (columns) of $\tilde{L}(t)$ are linearly independent of each other, but linearly dependent on the other rows (columns) of $\tilde{L}(t)$. Therefore, after removing these two rows and columns, $\tilde{L}_{f}(t)$ still has $2 N-2$ linearly independent rows (columns), and hence it has full rank.

The following Lemma will be important in proving the convergence of the follower forces to the leader's force.

Lemma 1: (Cauchy's interlacing theorem [19]) Let $B$ be a submatrix of $A=\left[\begin{array}{cc}B & y \\ y^{T} & a\end{array}\right]$, where $B$ is a Hermitian matrix in $\mathbb{R}^{N \times N}, A \in \mathbb{R}^{(N+1) \times(N+1)}, y \in \mathbb{R}^{N}$ and $a \in \mathbb{R}$. Then

$$
\begin{aligned}
\lambda_{1}(A) \leqslant \lambda_{1}(B) \leqslant & \lambda_{2}(A) \leqslant \cdots \\
& \leqslant \lambda_{N}(A) \leqslant \lambda_{N}(B) \leqslant \lambda_{N+1}(A) .
\end{aligned}
$$

Theorem 2: Under the centrosymmetric assumption (Assumption 1), all followers' forces in (20) will converge asymptotically to the leader's force $F_{1}$.

Proof: We first show that all the eigenvalues of $-\tilde{L}_{f}(t)$ are negative. By removing the first two rows and columns of $-\tilde{L}(t)$, we can apply Lemma 1 twice and get

$$
\begin{aligned}
& \lambda_{1}(-\tilde{L}(t)) \leqslant \lambda_{1}\left(-\tilde{L}_{f}(t)\right) \leqslant \cdots \cdots \\
& \quad \leqslant \lambda_{2 N-2}\left(-\tilde{L}_{f}(t)\right) \leqslant \lambda_{2 N-1}(-\tilde{L}(t))=0 .
\end{aligned}
$$

By Proposition 3, $\tilde{L}_{f}(t)$ has full rank, so $\tilde{L}_{f}(t) z=0$ has no nonzero solution. Thus we know $\lambda_{2 N-2}\left(-\tilde{L}_{f}(t)\right)<$ $\lambda_{2 N-1}(-\tilde{L}(t))=0$, that is, the largest eigenvalue of $\tilde{L}_{f}(t)$ is strictly less than zero.
Looking at (20), one can see that $F_{f}^{e q}=\left[F_{1}, F_{1}, \cdots, F_{1}\right]^{T}$ is an invariant equilibrium point, although the system is time-varying. Furthermore, since $\tilde{L}_{f}(t)$ has full rank, $\dot{F}_{f}=$ $-\tilde{L}_{f}(t) F_{f}-\tilde{L}_{f l}(t) F_{1}=0$ has an unique solution, such that $F_{f}^{e q}$ is the only equilibrium point. Then similarly to our technique in the proof of Theorem 1, we can decompose $F_{f}$ as

$$
F_{f}=F_{f}^{e q}+\delta .
$$

The dynamics of the disagreement can be obtained by

$$
\begin{aligned}
\dot{F}_{f}=\dot{\delta} & =\left(-\tilde{L}_{f}(t)\right)\left(F_{f}^{e q}+\delta\right)+\tilde{L}_{f l}(t) F_{1} \\
& =-\tilde{L}_{f}(t) \delta+\left(-\tilde{L}_{f}(t) F_{f}^{e q}+\tilde{L}_{f l}(t) F_{1}\right)=-\tilde{L}_{f}(t) \delta .
\end{aligned}
$$

Again, choose the Lyapunov function

$$
V=\frac{1}{2} \delta^{T} \delta
$$

and

$$
\begin{gathered}
\dot{V}=\delta^{T} \dot{\delta}=\delta^{T}\left(-\tilde{L}_{f}(t)\right) \delta \leqslant \lambda_{2 N-2}\left(-\tilde{L}_{f}(t)\right)\|\delta\|^{2}<0, \\
\ddot{V}=2 \delta^{T}\left(\tilde{L}_{f}(t)\right)^{2} \delta-\delta^{T} \dot{\tilde{L}}_{f}(t) \delta .
\end{gathered}
$$

Similarly, we have that $\ddot{V}$ is bounded using the same argument in Theorem 1. Then by Barbalat's, $\dot{V}=$ $\delta^{T}\left(-\tilde{L}_{f}(t)\right) \delta \rightarrow 0$, which implies $\delta \rightarrow 0$ since $\tilde{L}_{f}(t)$ has full rank. To sum up, the disagreement $\delta$ will vanish to zero asymptotically and all followers' forces will converge to the leader's force.

\section{Relaxing the Centrosymmetric Assumption}

In real applications, it would be difficult to precisely satisfy the centrosymmetric assumption (Assumption 1). It is reasonable to expect that robots will not know the shape of the object nor the attachment points of other robots. Furthermore, we may have an odd number of robots so centrosymmetry cannot be achieved. Our hope is that when the robots are close to a centrosymmetric distribution, the system will be robust enough to approximately reach a force consensus, especially when there exists a leader.

We model the deviation from the centrosymmetry as a perturbation. For an asymmetric attachment configuration $\left\{r_{1}, \cdots, r_{N}\right\}$, we can find a "nearest" centrosymmetric configuration $\left\{r_{1}^{\prime}, \cdots, r_{N}^{\prime}\right\}$ such that $\sum_{i}^{N}\left\|r_{i}-r_{i}^{\prime}\right\|$ is minimized. Then the leader-following dynamics (20) can be written in a perturbed form:

$$
\dot{F}_{f}=-\left(\tilde{L}_{f}(t)+\triangle_{f}(t)\right) F_{f}-\left(\tilde{L}_{f l}(t)+\Delta_{f l}(t)\right) F_{1},
$$

where $\Delta_{f}(t)$ and $\Delta_{f l}(t)$ are quantities induced by the centrifugal term in (7) and the asymmetry characterized by $\left(r_{i}-r_{i}^{\prime}\right)$. In addition, from (5) we know that $\Delta_{f}(t)$ and $\Delta_{f l}(t)$ are proportional to the angular velocity $\omega$ and acceleration $\alpha$ of the object. Therefore, if there is a way that we can stabilize the rotation of the object, for example, let the leader apply a feedback control torque $T_{1}$, then the perturbation from the asymmetry can be reduced.

Our claim is that if the perturbation $\Delta_{f}(t)$ and $\Delta_{f l}(t)$ is small enough, (21) is stable, and the followers' forces will be bounded in a region around the leader's force $F_{1}$. We will analyze this claim in more detail in future work. However, 
fx
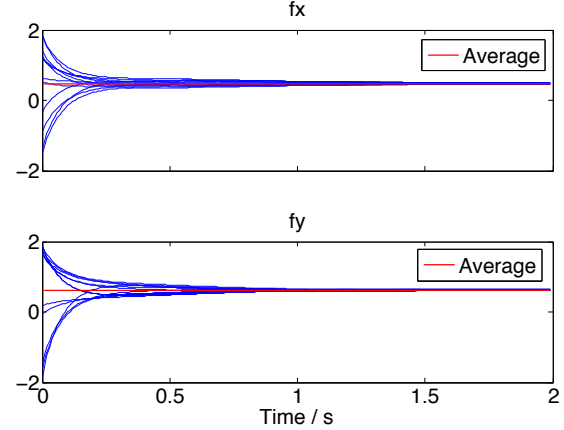

Fig. 3. Force consensus of 12 robots without leader.
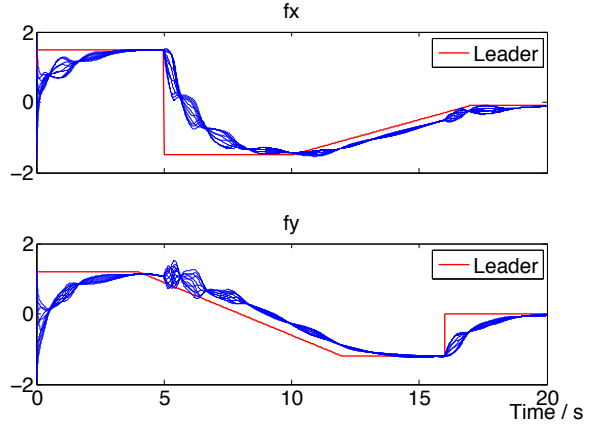

Fig. 4. Force following between 11 follower robots and one leader robot.

in the next section, we will show that even for a highly asymmetric distribution of robots, the robots' forces will still reach a consensus and follow a leader to manipulate an object. We use this to manipulate an inherently asymmetric grand piano with 1001 small simulated robots.

\section{Simulations}

In this section, we first show a numerical simulation of 12 robots to verify Theorems 1 and 2 . Then using the same settings for the robots and object, we also simulate a manipulation task in a physics simulator, where the robots are required to transport the object through a maze towards the destination. Performance comparison with our previous work is given to show the effect of using the motion measurements at different attachment points rather than at the center of mass of the object. Finally, we demonstrate a manipulation task for a grand piano with 1001 robots to verify our statement for the asymmetric case in the previous section.

Figure 3 demonstrates how the forces of 12 robots evolve over time without a leader. The object is a rectangle with dimension $0.6 \mathrm{~m} \times 0.3 \mathrm{~m}, M=1 \mathrm{~kg}$ and $J=0.09 \mathrm{~kg} \cdot \mathrm{m}^{2}$. Robots' attachment points are centrosymmetric around the center of the rectangle, as visualized in Figure 5. All the forces are initialized randomly and finally converge to the average after about $0.8 s$. Figure 4 shows the leader-following process, where we test the step and ramp control input of the leader. The followers' forces successfully follow the leader's, although with some delay and transient oscillation.

We also verify the numerical simulation above in a physics simulator called Open Dynamic Engine (ODE), as shown in Figure 5, where a leader robot actively changes its own force and torque according the object's relative pose to
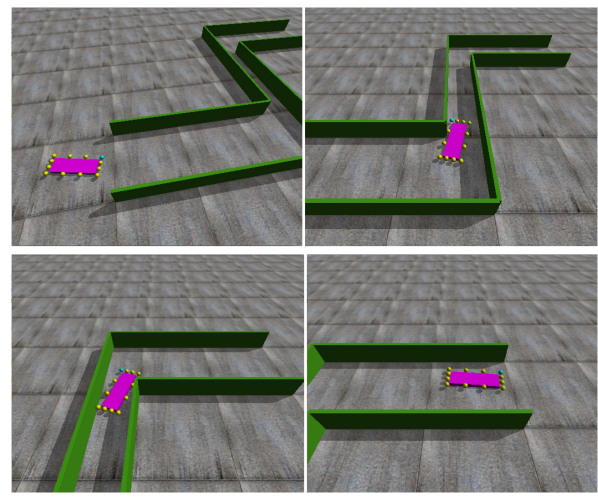

Fig. 5. Snapshots of transporting a rectangular object in ODE. The object is in purple. The follower and leader robots are denoted by yellow and blue spheres respectively.

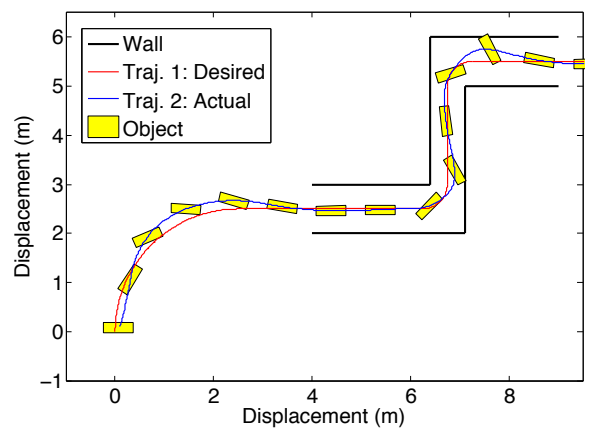

Fig. 6. Transporting rectangular object in ODE. Traj. (1) (red): desired trajectory. Traj. (2) (blue): actual trajectory where robots use local motion measurements. The leader also uses same PID parameters as Traj. (3) defined in Figure 7. The orientations of the object on the actual trajectory at different times are shown by the yellow rectangles.

the specified trajectory such that proper group force can be generated for the object to follow the trajectory. For the controller design for the leader robot, please refer to our previous work [1] for more details. Three trajectories, (2) (3) (4) under different setups are shown in Figure 6 and 7 while trajectory (1) is the desired reference trajectory. By comparing (2) (3) we can find that under the same PID parameters, the one using local motion measurements (2)) has worse performance (larger deviation from desired trajectory, larger angle overshoot) than the one using motion measurements at the center of mass (3)). This is because the term $R_{a}(t)$ in (11) tends to hinder the force consensus and create torque that will induce unwanted rotation. However, this problem can be fixed by choosing proper PID parameters for the leader robot. As Figure 7 suggests, the performance of using local motion measurements can be as good as that of using motion measurements at the center of mass after re-tuning the PID parameters.

Finally, we verify our statement in Section V by violating the centrosymmetric assumption. We challenge ourselves with the extreme situation. First of all, our target object is a grand piano, which is asymmetric itself. Secondly, we choose 1001 robots (an odd number) so that the centrosymmetric condition cannot be satisfied anyway. Lastly, we initialize the robots' positions randomly around the piano. 


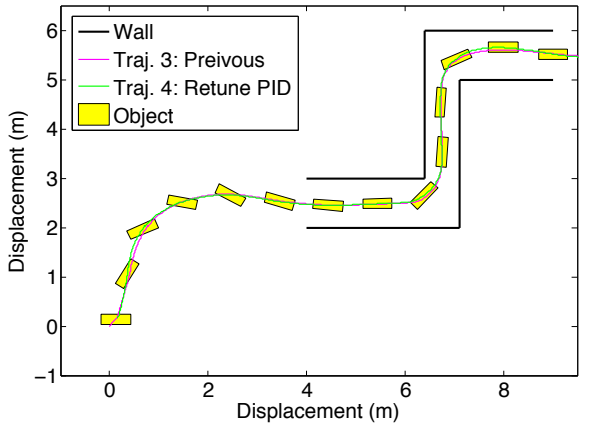

Fig. 7. Transporting rectangular object in ODE. Traj. (3) (purple): trajectory in our previous work [1] where robots use the the velocity and acceleration at the center of mass of the object. Traj. (4) (green): robots use local motion measurements but PID parameters are re-tuned to improve performance. The orientations of the object on the Traj. (4) at different times are shown by the yellow rectangles.
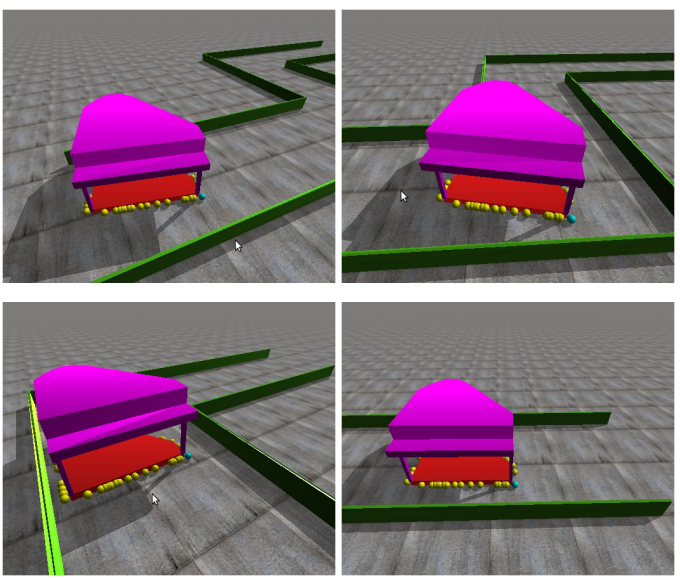

Fig. 8. Snapshots of transporting a grand piano through a maze with 1001 robots. The piano is mounted on a flat plate (in red) for the robots' convenience to grasp the piano and apply forces. The blue sphere denotes the leader robot while the yellow spheres are follower robots generated at random positions around the bottom of the piano. Due to the limit of space, only 50 out of 1001 robots are visualized.

Simulation shows that our algorithm works well, as shown in Figure 8, where the control objective is to move the piano through the maze while maintaining the angle of the object at 0 degree. Note that the dimension, $1.6 m(\mathrm{~L}) \times$ $1.5 m(\mathrm{~W}) \times 1 m(\mathrm{H})$, and weight $(290 \mathrm{~kg})$ of the simulated piano is the same as a real Yamaha $\mathrm{C} 1$ piano. All the simulation videos in this section can be found online at http://people.bu.edu/zjwang/cdc15.html

\section{CONCLUSION}

In this paper, we demonstrate an approach for a group of robots to collectively transport a large object without explicit communication. We prove that robots can reach a consensus on their forces by only using their local measurements of the motion of the object at their attachment points, if $\frac{M}{J} \sum_{i=1}^{N}\left\|r_{i}\right\|^{2}<N$ and robots are centrosymmetrically distributed around the object. Based on the consensus we also prove the convergence of the leader-following when there is a leader robot in the group. In our simulations, we show that one leader robot can successfully guide various numbers of robots to manipulate objects of different sizes.

In the future, we plan to work on the precise characterization of when centrosymmetry is broken, and give a rigorous proof of the consensus under asymmetric conditions. Also, we intend to implement our method on physical robots.

\section{REFERENCES}

[1] Z. Wang and M. Schwager, "Multi-robot manipulation without communication," in International Symposium on Distributed Autonomous Robotic Systems (DARS), 2014.

[2] R. Olfati-Saber and R. M. Murray, "Consensus problems in networks of agents with switching topology and time-delays," Automatic Control, IEEE Transactions on, vol. 49, no. 9, pp. 1520-1533, 2004.

[3] A. Jadbabaie, J. Lin, and A. S. Morse, "Coordination of groups of mobile autonomous agents using nearest neighbor rules," Automatic Control, IEEE Transactions on, vol. 48, no. 6, pp. 988-1001, 2003.

[4] Y. Hong, J. Hu, and L. Gao, "Tracking control for multi-agent consensus with an active leader and variable topology," Automatica, vol. 42, no. 7, pp. 1177-1182, 2006.

[5] H. G. Tanner, "On the controllability of nearest neighbor interconnections," in Decision and Control, 2004. CDC. 43rd IEEE Conference on, vol. 3. IEEE, 2004, pp. 2467-2472.

[6] Y. Zhang and Y.-P. Tian, "Consensus of data-sampled multi-agent systems with random communication delay and packet loss," Automatic Control, IEEE Transactions on, vol. 55, no. 4, pp. 939-943, 2010.

[7] T. Li and J.-F. Zhang, "Consensus conditions of multi-agent systems with time-varying topologies and stochastic communication noises," Automatic Control, IEEE Transactions on, vol. 55, no. 9, pp. 2043 2057, 2010

[8] H. McCreery and M. Breed, "Cooperative transport in ants: a review of proximate mechanisms," Insectes Sociaux, vol. 61, no. 2, pp. 99-110, 2014.

[9] O. Khatib, "A unified approach for motion and force control of robot manipulators: The operational space formulation," Robotics and Automation, IEEE Journal of, vol. 3, no. 1, pp. 43-53, 1987.

[10] D. Rus, B. Donald, and J. Jennings, "Moving furniture with teams of autonomous robots," in Intelligent Robots and Systems 95.'Human Robot Interaction and Cooperative Robots', Proceedings. 1995 IEEE/RSJ International Conference on, vol. 1. IEEE, 1995, pp. 235242.

[11] G. A. Pereira, M. F. Campos, and V. Kumar, "Decentralized algorithms for multi-robot manipulation via caging," The International Journal of Robotics Research, vol. 23, no. 7-8, pp. 783-795, 2004.

[12] W. Wan, R. Fukui, M. Shimosaka, T. Sato, and Y. Kuniyoshi, "Cooperative manipulation with least number of robots via robust caging," in Advanced Intelligent Mechatronics (AIM), 2012 IEEE/ASME International Conference on. IEEE, 2012, pp. 896-903.

[13] J. Fink, M. A. Hsieh, and V. Kumar, "Multi-robot manipulation via caging in environments with obstacles," in Robotics and Automation, 2008. ICRA 2008. IEEE International Conference on. IEEE, 2008, pp. 1471-1476.

[14] A. Becker, G. Habibi, J. Werfel, M. Rubenstein, and J. McLurkin, "Massive uniform manipulation: Controlling large populations of simple robots with a common input signal," in Intelligent Robots and Systems (IROS), 2013 IEEE/RSJ International Conference on. IEEE, 2013, pp. 520-527.

[15] G. Habibi, K. Zachary, W. Xie, M. Jellins, and J. McLurkin, "Distributed centroid estimation and motion controllers for collective transport by multi-robot systems," in Robotics and Automation (ICRA), 2015 IEEE International Conference on. IEEE, May 2015.

[16] J. Chen, M. Gauci, and R. Gross, "A strategy for transporting tall objects with a swarm of miniature mobile robots," in Robotics and Automation (ICRA), 2013 IEEE International Conference on, May 2013, pp. 863-869.

[17] S. Berman, Q. Lindsey, M. S. Sakar, V. Kumar, and S. Pratt, "Study of group food retrieval by ants as a model for multi-robot collective transport strategies." in Robotics: Science and Systems. Citeseer, 2010

[18] J. Fink, N. Michael, S. Kim, and V. Kumar, "Planning and control for cooperative manipulation and transportation with aerial robots," The International Journal of Robotics Research, vol. 30, no. 3, pp. 324-334, 2011.

[19] R. A. Horn and C. R. Johnson, Matrix analysis. Cambridge university press, 2012.

[20] M. Ji, A. Muhammad, and M. Egerstedt, "Leader-based multi-agent coordination: Controllability and optimal control," in American control conference, 2006, pp. 1358-1363. 